\title{
Transfer Characteristics of Three Kinds of Micro-groove Heat Pipes
}

\author{
Liu Yi-Bing ${ }^{\text {a }}$, Huang Zhi-Gang ${ }^{\text {a }}$
}

${ }^{a}$ Mechanical \& Electric Engineering Department, Shaoyang vocational \& technical college, Shaoyang, Hunan 422000, China

\begin{abstract}
In this paper, a simulation of three kinds of micro-groove plate heat pipes--- rectangular; trapezoidal and triangular, is conducted by thermal analysis software ANSYS. Through comparing with the focal point temperature value of the surfaces of micro-groove plate heat pipes, respectively being $30 \mathrm{~W}$; 40W; 50W, the result is obtained that trapezoidal plate heat piper has more excellent performance.
\end{abstract}

Index Terms: micro-channel plate heat pipe, ANSYS, modeling, grid division

(C) 2012 Published by MECS Publisher. Selection and/or peer review under responsibility of the Research Association of Modern Education and Computer Science.

\section{Instruction}

Cotter first proposed the theory of micro-heat pipe and its prospects ${ }^{\text {[11 }}$ in Fifth International Conference on heat pipe which was held in Japan in 1984. Since vapor flow channels within micro-channel plate heat pipe are connected with each other, the interface friction between liquid and vapor is reduced, resulting in improving heat transfer performance. At present, micro-channel plate heat pipe has become the focus of research and development.

Peterson and others ${ }^{\mathbf{2}}$ made the theoretical analysis of the triangular flat heat pipe, which confirmed the minimum meniscus radius on evaporator section of triangular channel and maximum heat capacity of micro heat pipe. Faghri A., etc. ${ }^{\mathbf{3} 1}$ carried out experimental and theoretical analysis for trapezoidal and rectangular copper micro-channels - water heat pipes and they agreed that heat pipe with large aspect ratio of grooves has better heat transfer performance and they also made an analytical reasoning to its capillary limit. Some comparative experimental studies on three different aspect ratio micro-groove heat pipe flat conducted by Fan Chunli, etc. ${ }^{\mathbf{4} \mathbf{l}}$ show that flat heat pipe whose aspect radio is high has better heat transfer performance. However, the comparative study on heat transfer performance of plate heat pipe with different channel structure is not deep enough. Based on the comparison of triangular; rectangular and trapezoidal plates of 
three different micro-groove heat pipe structure made by thermal analysis software ANSYS, the conclusion is: the trapezoid structure of the flat heat pipe has the best heat transfer characteristics, followed by rectangular structure, the last is triangular structure.

\section{Three micro channel plate heat pipe structures}

The dimensions of flat heat pipe used in the experiments are $60 \mathrm{~mm} * 22 \mathrm{~mm} * 5 \mathrm{~mm}$; thickness of top to bottom cover is $0.5 \mathrm{~mm}$; thickness of the side wall is $1 \mathrm{~mm}$, all of which meet the strength requirements. The second distilled water is used as working media. In heat pipe, the length of evaporator section; condenser section and adiabatic section are $20 \mathrm{~mm}$, oxygen-free copper tube materials are used, the internal channel is 10 channels connected with each other to reduce the interface friction of the reverse movement of steam and liquid, the channel structures are triangular, rectangular and trapezoidal. Cross-section shown in Figure 1:
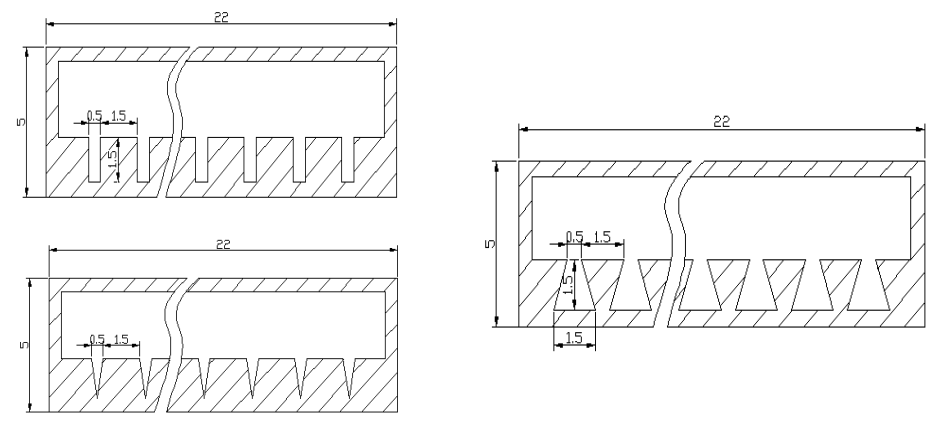

Fig 1 Cross-section of three kinds of micro-groove plate heat pipe

\section{ANSYS Thermal Analysis}

ANSYS is the first international popular software recognized by ISO9001 quality certification and a large general-purpose finite element analysis software which set structure; heat flow; electromagnetic and acoustic in one ${ }^{151}$ Analysis process characterized by modeling simple; fast and conveniently is divided into preprocessing; loading solution and post-processing is , in which modeling and analysis of meshing is the key technology. In the micro channel plate heat pipe simulation, the following assumptions are made: (1) refrigerant vapor is saturated ideal gas; (2) start performance and 30 seconds to reach steady state; the ambient temperature and the boundary conditions do not change over time; (3) heat contact surface is boundary heat flux density; (4) ignored components of the contact resistance between the contact surfaces; (5) material uniformly continuous; (6) only consider the axial heat pipe cooling.

\subsection{Geometric Modeling}

Used in the pro / $\mathrm{E}$ on the model, and then imported into ANSYS, the three pro / $\mathrm{E}$ three-dimensional geometric models shown in Figure 2, select the eight-node thermal analysis unit hexahedral element thermal, SOLID70. 

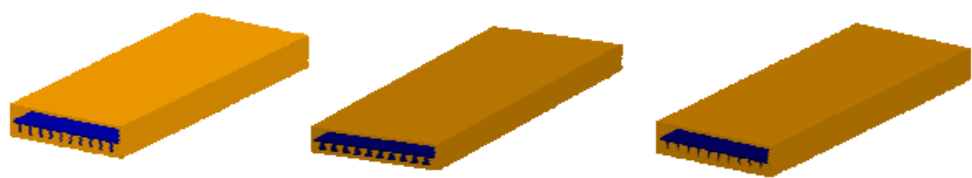

Fig 2 three-dimensional geometrical model of three kinds of micro-groove plate heat pipes

\section{2. mesh}

As ICEM CFD Tetra tetrahedral mesh device has a powerful mesh smoothing algorithm, and the local adaptation algorithm for encryption and rough, so by the use of Tetra8 tree algorithm, the volume is filled with tetrahedral prism and tetrahedral hybrid mesh ( free grid) is generated. Three different structural mesh flat heat pipes as shown in Figure 3

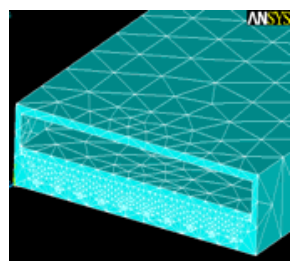

(node 4302, unit 542

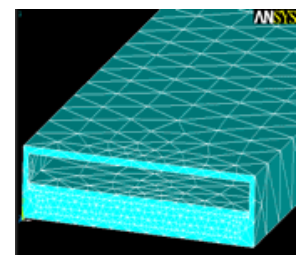

(node 4318, unit 546 )

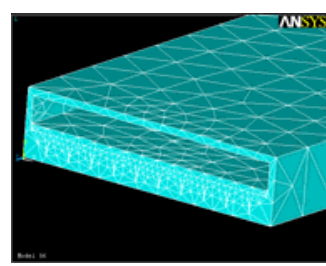

(node 4297, unit 538)

Fig 3.Grid division of three kinds of micro-groove plate heat pipes

\subsection{Load Solution}

Using ANSYS / Multi physics and Sparse multi-physics solver, Newton-Raphson algorithm solves the transient temperature field. Specify the initial time step $\triangle t=35$; the minimum time step 30; the maximum time step 200; the automatic time step chosen on. Taking into account both accuracy and stability, using the Euler backward difference method, first order transient integration parameter is set to THETA $=0.75$; vibration limit LimitOSLM $=0.5$; tolerance $\mathrm{TOL}=0.1$; loads of bands style, that is, all containing loads are put on child in the first load step, and the rest remains the same. To speed up, solving the Line Search for the difference is ON. To this end, respectively, set the heating power (load) for the $30 \mathrm{~W} ; 40 \mathrm{~W}$; $50 \mathrm{~W}$ load to solve three cases, the heating power of 30W, for example, after loading the model shown in Figure 4:
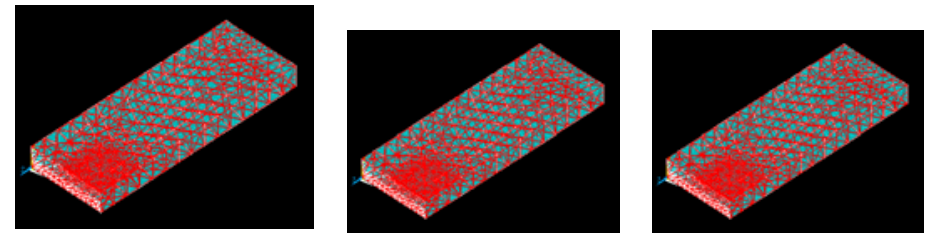

Fig 4.Modeling of three kinds of micro-groove heat pipes after loading 30W

\section{4. post-processing}

Using POST1, draw the temperature cloud and the temperature vector when it is 35 seconds and heating power is 30W, as shown in Figure 5, Figure 6 

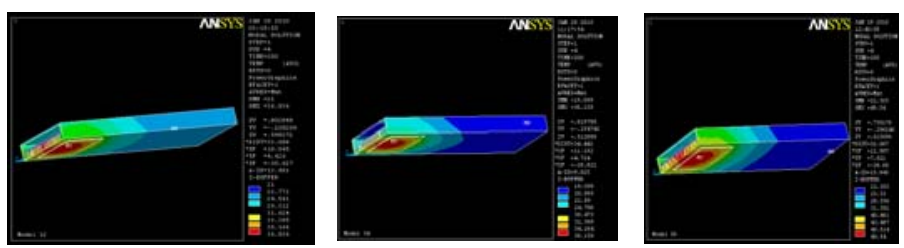

Fig 5 Temperature cloud of three kinds of micro-groove heat pipes
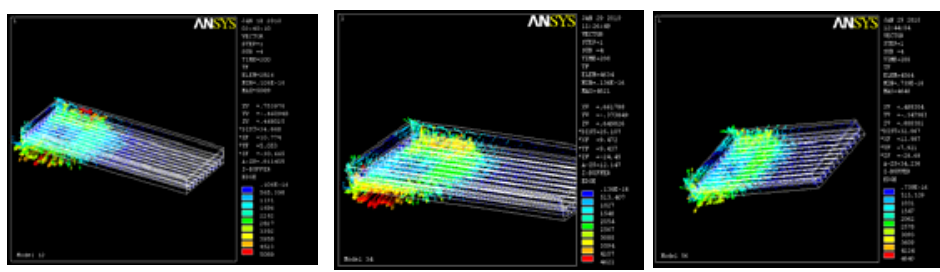

Fig 6 Temperature vector of three kinds of micro-groove heat pipes

\subsection{Simulation results and analysis}

In this paper, the Simulation is under the condition of at room temperature $18{ }^{\circ} \mathrm{C}$; filling rate of 1.3 ; horizontal; running for 30 seconds after the heat pipe thermal equilibrium. The test point being the center of the lower surface of simulated heat pipe, the temperature of the lower surface center is extracted by the command * GET; Par; Entity; ENTNUM; Item1; IT1NUM; Item2; IT2NUM and when the heating power was $30 \mathrm{~W}$; 40W; 50W; the test point temperature versus time curve in three different micro-groove structure is plotted by Post26. Shown in Figure 7, Figure 8, Figure 9:
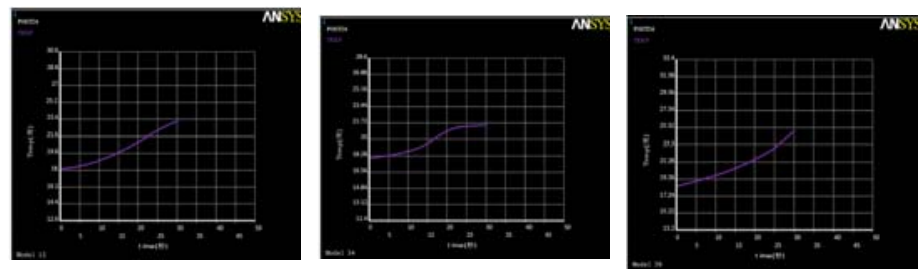

Fig 7 Curve of test point temperature versus time under 30W load
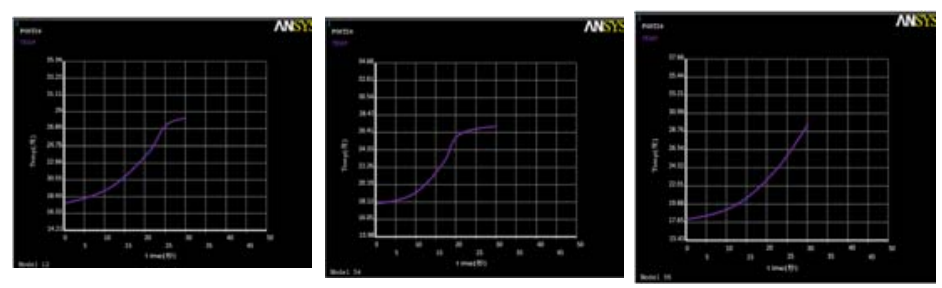

Fig 8 Curve of test point temperature versus time under $40 \mathrm{~W}$ load 

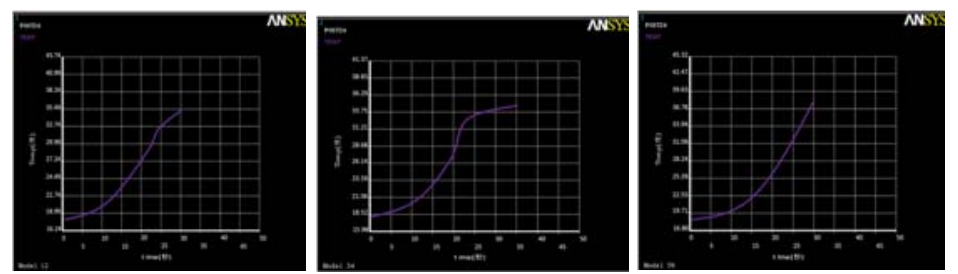

Fig 9 Curve of test point temperature versus time under 50W load

Table 1. Test point temperature under different heating power of three kinds of micro-groove heat pipes

\begin{tabular}{|l|l|l|l|}
\hline Heating power & Rectangular & trapezoid & triangular \\
\hline $30 \mathrm{~W}$ & $23.272^{\circ} \mathrm{C}$ & $21.662^{\circ} \mathrm{C}$ & $25.126^{\circ} \mathrm{C}$ \\
\hline $40 \mathrm{~W}$ & $27.863^{\circ} \mathrm{C}$ & $27.012^{\circ} \mathrm{C}$ & $29.422^{\circ} \mathrm{C}$ \\
\hline $50 \mathrm{~W}$ & $35.426^{\circ} \mathrm{C}$ & $24.122^{\circ} \mathrm{C}$ & $37.474^{\circ} \mathrm{C}$ \\
\hline
\end{tabular}

By comparing the temperature of all test points, we can see that the trapezoid structure of the flat heat pipe has the best heat transfer characteristic; followed by rectangular structure; the last is triangular structure. The reason may be in trapezoidal structure, under capillary force, the thin liquid film evaporation zone whose heat transfer area is the strongest in the region is relatively elongated, making the heat transfer better performance.

\section{4. Conclusion}

In this paper, using ANSYS thermal analysis software and doing contrasting simulation of three kinds of micro-groove structure channel plate heat pipe, we can draw the following conclusions:

(1)Among the heat transfer performances of three micro-groove structure, the trapezoid structure of the flat heat pipe has the best heat transfer characteristics, followed by rectangular structure, the last is triangular structure.

(2)It is a new research method to simulate and compare the heat transfer performances of micro channel plate in different structure, which may be unsatisfactory and also need improving in the future study.

\section{Acknowledgements}

Fund Project: Hunan Provincial Department of Education research project (08D110), Shaoyang Municipal Science and Technology Program (08SC016) funded

\section{References}

[1] Liu Yi-Bing, electronic cooling technique [J], Electronics Process Technology, 2007,28 (5) :286-289

[2] Peterson GP, MaHB、Theoretical analysis of the maximum heat transport in triangular grooves:a study of idealized micro heat pipes[J], ASME Journal of Heat Transfer , 1996, 118: 731-739

[3] HopkinsR, Faghri A, Khrustalev D, Flat Miniature Heat Pipes with Micro Capollary Grooves[J], ASME Journal of Heat Transfer , 1999, 121: 102-109

[4] Fan-Chun Li, Qu Wei, Sun F., etc., three micro-groove structure of the flat heat pipe heat transfer 
experimental study [J], electronic devices, 2003,26 (4) :357-360

[5] Liu Yi-Bing, Liu Guohua, ANSYS thermal analysis of the key technologies and research [J], Chongqing Institute of Technology (Natural Science), 2008,10 (6) :104-107 